hep-ph/0304256

\title{
Rationalizing right-handed neutrinos
}

\author{
Graham D. Kribs \\ Department of Physics, University of Wisconsin, Madison, WI 53706 \\ kribs@physics.wisc.edu
}

\begin{abstract}
A simple argument based on an $\mathrm{SU}(3)$ gauged horizontal symmetry is presented that connects the explanation for three generations of matter with the existence of a triplet of right-handed neutrinos. This rationale for right-handed neutrinos is analogous to, but completely independent of, grand unification or extra universal dimensions. A brief discussion of the supersymmetrized SU(3) model is also given, pointing out that certain problems in ordinary supersymmetric models such as fast proton decay via dimension-5 Planck-suppressed operators can be naturally solved.
\end{abstract}

One of the most remarkable features of the Standard Model (SM) is that matter fermions are chiral and yet all gauge [1, 2] and gravitational [3] anomalies vanish for each generation. A known but not often emphasized fact about the matter content is that, given one generation with unfixed hypercharges, anomaly cancellation determines the relative hypercharge assignment to be precisely what has been established by experiment [4]. In other words, electric charge quantization is essentially automatic without grand unification. This fact, taken at face value, is circumstantial evidence against the existence of right-handed neutrinos. By definition a candidate for a right-handed neutrino is any fermion that is uncharged under all of the SM gauge symmetries. Yet, gauge symmetries are precisely the reason that each type of matter $(Q, u, d, L, e)$ is tied with the other matter fields together in a self-consistent, exclusive fashion. In addition, non-chiral matter allows a new mass scale unconnected to electroweak symmetry breaking that only further complicates our understanding of mass generation and mass hierarchies. Extensions of the SM with non-chiral matter, such as adding right-handed neutrinos, therefore appear to be contrary to all of the guiding wisdom gleaned from experiment, at least until recently. (Those who are still in doubt need only observe the agony that the $\mu$ problem causes avatars of supersymmetry.)

Neutrino experiments [ 5, 6, 7], however, have firmly established that the neutrinos oscillate between each generation and thus they have mass. The largest mass of any one neutrino is constrained to be less than about 2 $\mathrm{eV}$ [8], and more likely their mass is one to a few orders of magnitude below this, depending on the generation. The mechanism of mass generation for neutrinos remains a mystery. If neutrinos acquire mass analogously to the SM matter fermions, preserving lepton number, then the particle content must be extended with at least two right-handed neutrinos $\nu_{1,2}$. Ordinary Yukawa terms $\mathcal{L}=\lambda_{\nu} L H \nu^{c}$ with tiny couplings $\lambda_{\nu} \lesssim 10^{-12}$ suffice to explain the two undisputed mass differences found in neutrino oscillation experiments.

But, the global symmetry behind lepton number conservation is not expected to be exact. At dimension-5, the operator $H L H L / M$ violates lepton number by two units and leads to a tiny Majorana mass $v^{2} / M$ for left-handed neutrinos. This transmutes the neutrino mass hierarchy problem from explaining $\lambda_{\nu} \lesssim 10^{-12}$ to instead explaining $v / M \lesssim 10^{-12}$. To embrace the dimension-5 neutrino mass explanation means the SM effective theory breaks down at $M \lesssim 10^{14} \mathrm{GeV}$. This is somewhat disconcerting since there are dimension- 6 operators that violate lepton and baryon number, leading to a proton decay rate that is excluded by experiment unless $M \gtrsim 10^{16} \mathrm{GeV}$. Hence, while lepton number must be be violated at $M$ to explain neutrino masses, baryon number must be preserved to keep the proton stable. The simplest phenomenological explanation for lepton number violation without baryon number violation at the cutoff scale $M$ is to add righthanded neutrinos to the SM with ordinary Yukawa couplings, plus a heavy Majorana mass term $\mathcal{L}=M \nu^{c} \nu$ for the right-handed neutrinos. The resulting combination of a Dirac mass and a heavy Majorana mass leads to the famous "see-saw" neutrino mass matrix [ $]$

$$
\left(\begin{array}{cc}
0 & \lambda_{\nu} v \\
\lambda_{\nu} v & M
\end{array}\right)
$$

Diagonalizing this mass matrix, or equivalently integrating out the right-handed neutrino gives back the SM plus the dimension-5 operator $H L H L / M$ with a welldefined coefficient, $\lambda_{\nu}^{2}$, and thus predicted neutrino mass, $\lambda_{\nu}^{2} v^{2} / M$. A right-handed neutrino with Majorana mass $M$ therefore provides an ultraviolet completion of the effective theory beyond the cutoff $M$, explaining why only lepton number was violated at $M$.

The difficulty with such neutrino mass generation mechanisms is that they do not really solve the neutrino mass hierarchy problem, and worse still, require precisely those odd-ball fields - right-handed neutrinos - that are unconnected to SM matter through gauge anomalies. Furthermore, the see-saw explanation requires a new Majorana mass scale unconnected with electroweak symmetry breaking. These facts would ordinarily be highly distressing except for a remarkable coincidence: the scale $M \sim 10^{14} \mathrm{GeV}$ is tantalizingly close to where the SM gauge couplings come to an approximate intersection. Such an intersection is predicted by grand unified theories (GUTs), providing justification for the new scale. 
Furthermore, in an SO(10) GUT each right-handed neutrino is elegantly fused with each generation of SM matter into a single 16 representation [10]. This is really just an artifact of unifying into a GUT group with rank greater than that of the SM, since candidates for righthanded neutrinos in GUTs are those fields uncharged under the SM symmetries but charged under some additional gauge symmetry [for $\mathrm{SO}(10)$ this is the extra $\mathrm{U}(1)$ under the decomposition $\mathrm{SO}(10) \rightarrow \mathrm{SU}(5) \times \mathrm{U}(1)$ ]. Rank $>4$ GUTs therefore provide a rationale for $n=0$ mod 3 right-handed neutrinos whenever each generation is unified into a single representation of the group.

Unfortunately, grand unification has many well-known problems of implementation. Non-supersymmetric grand unification proposals suffer from the hierarchy problem as well as a rather inexact unification of gauge couplings. Both non-supersymmetric and supersymmetric unification models predict proton decay at a rate that has been experimentally ruled out in the simplest models. Also, several theoretical problems pervade unification ranging from understanding how the Higgs is embedded into a GUT representation (the doublet-triplet splitting problem), how (or if) Yukawa couplings are unified, etc. Such experimental and theoretical problems ought to induce us to reconsider GUTs as the origin of right-handed neutrinos and the Majorana mass scale. Is there any rationale independent of unification that predicts righthanded neutrinos as well as the Majorana mass scale?

Suppose the explanation for the number of generations is that each field's three generations $\left(Q_{1,2,3}, u_{1,2,3}\right.$, etc.) correspond to three components of a multiplet of a "horizontal" flavor symmetry. There are only two continuous symmetries that are suitable for this purpose possessing a 3 representation: $\mathrm{SU}(3)$ 11] and $\mathrm{SU}(2) \sim \mathrm{SO}(3)$ 12, 13]. $\mathrm{SU}(2)$ can be summarily dismissed if right-handed neutrinos are required to be in a chiral representation of the new symmetry. It has already been emphasized that nonchiral fermions, and right-handed neutrinos in particular, seem to have no (aesthetic) place in the SM if anomaly cancellation is to connect all matter together. There is no hope with $\mathrm{SU}(2)$ since it is anomaly-free. $\mathrm{SU}(2)$ also does not predict the number of generations since representations of any dimension are possible 14. Instead, SU(3) admits chiral fermions with only certain dimensionality - there can be three but not two, four, five, seven, etc. generations. Moreover, SU(3) provides two additional key ingredients: (1) there is an additional anomaly cancellation condition on the matter content if $\mathrm{SU}(3)$ is at least weakly gauged, and (2) all fermion masses, including right-handed neutrino masses, arise from spontaneous symmetry breaking. Before proceeding, note that the connection between SU(3) anomaly cancellation and the existence of right-handed neutrinos was made some time ago in 11]. In this paper the argument in presented in detail, contrasting with grand unification and universal extra dimensions, and then implications for a supersym- metrized version are briefly discussed.

Gauging a new symmetry in which SM fermions transform is non-trivial and requires the cancellation of all gauge anomalies associated with the new symmetry. There are potentially eight new gauge anomalies associated with $\mathrm{SU}(3)_{f}:\left[\mathrm{SU}(3)_{f}\right]^{3} ; \mathrm{SU}(3)_{f} \times\left[\mathrm{SU}(3)_{c}\right]^{2}$; $\left[\mathrm{SU}(3)_{f}\right]^{2} \times \mathrm{SU}(3)_{c} ; \mathrm{SU}(3)_{f} \times\left[\mathrm{SU}(2)_{L}\right]^{2} ;\left[\mathrm{SU}(3)_{f}\right]^{2} \times$ $\mathrm{SU}(2)_{L} ; \mathrm{SU}(3)_{f} \times\left[\mathrm{U}(1)_{Y}\right]^{2} ;\left[\mathrm{SU}(3)_{f}\right]^{2} \times \mathrm{U}(1)_{Y} ;$ and $\mathrm{SU}(3)_{f} \times[\mathrm{grav}]^{2}$. Six of these are trivially zero since $\operatorname{tr}\left[t^{a}\right]=0$ for $\mathrm{SU}(\mathrm{N})$ gauge groups with $N>1$. This leaves the mixed flavor symmetry/hypercharge anomaly $\left[\mathrm{SU}(3)_{f}\right]^{2} \times \mathrm{U}(1)_{Y}$, and the $\left[\mathrm{SU}(3)_{f}\right]^{3}$ anomaly. The mixed anomaly leads to a condition on the sum of the hypercharges of the SM fermions that is equivalent to the mixed $[\mathrm{grav}]^{2} \times \mathrm{U}(1)_{Y}$ anomaly

$\left[S U(3)_{f}\right]^{2} \times U(1)_{Y}:\left(6 Y_{Q}+3 Y_{u}+3 Y_{d}+2 Y_{L}+Y_{e}\right)=0$,

and so automatically cancels. The $\left[\mathrm{SU}(3)_{f}\right]^{3}$ anomaly, however, does not cancel with just the SM fermion content 11,12$]$.

This is straightforward to see: Five types of matter $(Q$, $u, d, L, e)$ can be assigned to either $\mathbf{3}$ or $\overline{\mathbf{3}}$ representations. Two of the five fields contribute an even number of 3's or $\overline{3}$ 's to the anomaly $(Q: \pm 6 ; L: \pm 2)$ while the remaining three fields contribute an odd number of 3 's or $\overline{3}$ 's $(u: \pm 3, d: \pm 3, e: \pm 1)$. The sum of two even numbers and three odd numbers is an odd number, and so $\left[\mathrm{SU}(3)_{f}\right]^{3}$ anomaly $a \propto n_{\mathbf{3}}-n_{\overline{\mathbf{3}}}$ cannot be canceled no matter how SM matter is assigned to $\mathrm{SU}(3)_{f}$.

The simplest assignment of matter in $\mathbf{3}$ or $\overline{\mathbf{3}}$ representations allows ordinary Yukawa couplings. Here the Higgs scalar doublet is assumed to be a singlet under $\mathrm{SU}(3)_{f}$, since there is no need for (and various reasons that disfavor) more than one Higgs doublet in the SM. Gauge invariance of the three Yukawa couplings of the SM implies three relations among the anomaly coefficients of SM matter

$$
\begin{gathered}
Q H u^{c} \Rightarrow a(Q)+a\left(u^{c}\right)=0 \\
Q H^{*} d^{c} \Rightarrow a(Q)+a\left(d^{c}\right)=0 \\
L H^{*} e^{c} \Rightarrow a(L)+a\left(e^{c}\right)=0
\end{gathered}
$$

Without loss of generality $Q$ can be chosen to be a $\mathbf{3}$, then $u^{c}$ and $d^{c}$ must both be $\overline{\mathbf{3}}$ 's. There are two choices for the leptons: $\left[L(\mathbf{3}), e^{c}(\overline{\mathbf{3}})\right]$ or $\left[L(\overline{\mathbf{3}}), e^{c}(\mathbf{3})\right]$. In either case, the $\left[\mathrm{SU}(3)_{f}\right]^{3}$ anomaly coefficient becomes

$$
a=6-3-3 \pm(2-1)= \pm 1 \text {. }
$$

Notice that the anomaly associated with colored fermions self-cancels, but with the leptons it does not cancel regardless of assigning $\left(L, e^{c}\right)$ into a $(\mathbf{3}, \overline{\mathbf{3}})$ or $(\overline{\mathbf{3}}, \mathbf{3})$.

Intriguingly, the $\left[\mathrm{SU}(3)_{f}\right]^{3}$ anomaly is canceled by adding a single new field that transforms as a $\overline{\mathbf{3}}$ [for $L(\mathbf{3}), e^{c}(\overline{\mathbf{3}})$ ] or $\mathbf{3}$ [for $L(\overline{\mathbf{3}}), e^{c}(\mathbf{3})$ ] under $\mathrm{SU}(3)_{f}$. To avoid spoiling the SM anomaly cancellation conditions 
this field must be neutral under SM gauge symmetries. Hence, this anomaly-cancellation field has precisely the quantum numbers of a right-handed neutrino. Also, a Yukawa interaction connecting the left-handed with the right-handed neutrino, $\mathcal{L}=L H \nu^{c}$, is automatically allowed by $\mathrm{SU}(3)_{f}$ gauge invariance regardless of the initial choice of $(\mathbf{3}, \overline{\mathbf{3}}, \overline{\mathbf{3}})$ versus $(\overline{\mathbf{3}}, \mathbf{3}, \mathbf{3})$ for $\left(L, e^{c}, \nu^{c}\right)$.

This is a remarkable result. Let me restate the assumptions and the implication: Assuming (1) the explanation for the number of generations is a gauged $\mathrm{SU}(3)_{f}$ flavor symmetry, (2) all matter is assigned to chiral representations $(\mathbf{3}$ or $\overline{\mathbf{3}})$ of $\mathrm{SU}(3)_{f}$, and (3) ordinary Yukawa couplings are $\mathrm{SU}(3)_{f}$ gauge invariant, then there must exist one set of right-handed neutrinos $\nu_{1,2,3}$ transforming as a triplet of $\mathrm{SU}(3)_{f}$.

It is important to emphasize that this flavor symmetry rationale for right-handed neutrinos is completely independent of grand unification. In fact, the simplest assignment that allows Yukawa couplings to be gauge invariant under $\mathrm{SU}(3)_{f}$ does not commute with the usual matter embeddings in unified representations of GUTs. For example, $\mathrm{SU}(5)$ [as well as $\mathrm{SO}(10)$ and $E_{6}$ ] unifies $Q$ and $u$ into a single representation; this is inconsistent with the $\mathrm{SU}(3)_{f}$ assignment given above. However, Yukawa couplings are notoriously over-constrained in GUTs as well as flavor symmetry models. SU(5) predicts the down and lepton Yukawas of each generation should unify, and SO(10) predicts up, down, and lepton Yukawas to unify. These predictions are badly broken at low energies, and not much better at the GUT scale for all but perhaps $\lambda_{b}$ and $\lambda_{\tau}$. Analogously, the simplest $\mathrm{SU}(3)_{f}$ assignment allows Yukawa couplings for all generations, but no generational differences. This must come from additional structure related to the flavor symmetry breaking that has not been specified here. Nevertheless, the matter (and Higgs) assignments under $\mathrm{SU}(3)_{f}$ can be suitably modified to commute with grand unification. This was done in several early works on gauged $\mathrm{SU}(3)_{f} \times \mathrm{SU}(5)$ 15]. There they found that many more triplets (or perhaps larger representations) of right-handed neutrinos were needed to cancel the $\left[\mathrm{SU}(3)_{f}\right]^{3}$ anomaly. For the purposes of this paper, it is enough to observe that there must be at least one triplet of right-handed neutrinos to gauge $\mathrm{SU}(3)_{f}$.

The absence of a signal for new physics in flavorchanging neutral current processes places an important constraint on the scale of $\mathrm{SU}(3)_{f}$ symmetry breaking. The constraint arises from the tree-level exchange of flavor gauge bosons that lead to transitions between samecharge, different-generation quarks or leptons. Integrating out heavy flavor gauge bosons results in a low-energy effective theory with new contributions to four-fermion, flavor-violating operators

$$
\frac{g_{f}^{4}}{M_{f}^{2}}\left(\bar{f}_{i} \gamma_{\mu} f_{i}\right)\left(\bar{f}_{j} \gamma^{\mu} f_{j}\right)
$$

where $g_{f}$ is the $\mathrm{SU}(3)_{f}$ gauge coupling and $M_{f}$ is the symmetry breaking scale. If the couplings are CP-conserving, one of the strongest constraints comes from the $\Delta s=2$ process that contributes to the $K_{0}-\bar{K}_{0}$ mass difference. Estimates of the bound on the four-quark operator suggest $M_{f} \gtrsim g_{f}^{2} \times 1600 \mathrm{TeV}$ [16]. The bound is significantly stronger if the couplings maximally violate $\mathrm{CP}$. In any case, for a flavor gauge coupling that is of order the SM gauge couplings, the bound on the symmetry breaking scale is at least hundreds of $\mathrm{TeV}$. This is reminiscent of the constraints on extended technicolor [17].

The benefit of right-handed neutrinos transforming under a chiral representation of the flavor symmetry is that the Majorana mass scale is no longer arbitrary. The right-handed neutrino Majorana mass is generated through flavor symmetry breaking, analogous to SM fermion masses generated through electroweak symmetry breaking. The scale $M_{f}$ is not predicted, but obviously there is no conflict between the lower bound $M_{f} \gtrsim 1000 \mathrm{TeV}$ from flavor-changing constraints and the upper bound $M_{f} \lesssim 10^{14} \mathrm{GeV}$ needed for a successful see-saw explanation of neutrino masses. If $M_{f}$ were near the lower bound, future experiments could search for deviations from (or as-yet unobserved) flavor-changing neutral current processes as a signal for $\mathrm{SU}(3)_{f}$. This would require neutrino Yukawa couplings $\lambda_{\nu} \sim 10^{-4}$ nearer in value to their lepton cousins.

How are right-handed neutrino Majorana masses generated from flavor symmetry breaking? Consider a pair of complex scalar fields in the fundamental representation $\Sigma_{1,2}(\mathbf{3})$ that acquire unaligned vacuum expectation values. This is sufficient to break $\mathrm{SU}(3)_{f} \rightarrow$ nothing. A right-handed neutrino mass arises from the dimension-4 operator $\epsilon_{i j k} \nu_{i}^{c} \nu_{j} \Sigma_{k}^{*}$ replacing $\Sigma$ by its vev. Curiously, this two-field breaking model gives mass to just two offdiagonal components of the $3 \times 3$ Majorana mass matrix in flavor space

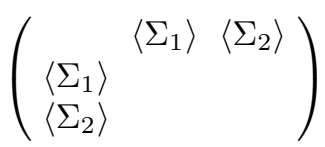

due to the anti-symmetric contraction of $\mathrm{SU}(3)_{f}$ indices. This may be a useful starting point for generating an interesting neutrino mass texture. Also, the flavor symmetry could be broken in stages, such as $\mathrm{SU}(3) \rightarrow \mathrm{SU}(2)$ $\rightarrow$ nothing, that may be similarly useful for quark, lepton, or neutrino mass textures.

$\mathrm{SU}(3)_{f}$ is not the only rationale for three generations and three right-handed neutrinos. In a recent proposal called "universal extra dimensions" (UED) [18], all matter, Higgs, and gauge bosons are promoted to six dimensional fields, and the more complicated gauge and gravitational anomaly structure of six dimensional theories is used to constrain the matter content [19]. Ref. 19] found that cancellation of the global gauge anomaly 20] required the number of generations to be $n_{g}=0 \bmod$ 
3 , and cancellation of the pure gravitational anomaly required $n=n_{g}$ fermionic fields uncharged under the SM gauge group. This is intriguingly similar to the $\mathrm{SU}(3)_{f}$ symmetry argument, since the matter content is similarly restricted by anomaly cancellation of a larger symmetry structure. Other similarities are remarkable: 19] required that all matter was chiral in $6-\mathrm{D}$, analogous to requiring all matter to be in chiral representations of $\mathrm{SU}(3)_{f}$. This led to two possible chirality assignments in UED that are precisely analogous to the $\mathbf{3}$ versus $\overline{\mathbf{3}}$ "chirality" possibilities for the SM fermions under $\mathrm{SU}(3)_{f}$. Specifically, the quark doublet $(Q)$ must have the opposite chirality to the quark singlets $(u, d)$, and the lepton doublet $(L)$ must have the opposite chirality to the lepton singlets $(e, \nu)$. In UED the lepton doublet could have the same or the opposite chirality of the quark doublet, just as here the lepton doublet could be assigned to the same $(\mathbf{3})$ or opposite $(\overline{\mathbf{3}})$ representation of the quark doublet. Finally, the UED rationale for three generations and three right-handed neutrinos does not depend on the compactification scale, just as the $\mathrm{SU}(3)_{f}$ argument does not depend on the flavor symmetry breaking scale.

There are a few important differences between the six dimensional UED model and the $\mathrm{SU}(3)_{f}$ model. The higher dimensional nature of UED implies there is an effective theory cutoff scale that is only an order of magnitude above the compactification scale; in the $\mathrm{SU}(3)_{f}$ model, there is no such restriction. Several gauge anomalies, such as $\left[\mathrm{SU}(2)_{L}\right]^{2} \mathrm{U}(1)_{Y}$ that are automatically canceled in the $\mathrm{SU}(3)_{f}$ model, are canceled in UED only via the Green-Schwarz mechanism with additional matter. Finally, the prediction of three generations is not easily extended to a supersymmetric six-dimensional "universal" model for a variety of reasons [19], whereas the $\mathrm{SU}(3)_{f}$ model can be quite simply supersymmetrized as will be briefly sketched below.

Everything that has been said for the SM with a gauged $\mathrm{SU}(3)_{f}$ flavor symmetry also applies to a straightforward extension of the minimal supersymmetric standard model (MSSM). This means promoting matter supermultiplets to (anti-)fundamental representations of $\mathrm{SU}(3)_{f}$ while the Higgs supermultiplets remain singlets, in exact analogy with the non-supersymmetric case. (In the following discussion the same notation is used for the MSSM chiral superfields as for the SM fermion fields.) There are, however, new restrictions on the allowed operators in the supersymmetrized $\mathrm{SU}(3)_{f}$ model. The most interesting, model-independent restriction is that the dimension-5 operators leading to proton decay

$$
\frac{Q Q Q L}{M_{\mathrm{Pl}}}, \frac{u^{c} u^{c} d^{c} e^{c}}{M_{\mathrm{Pl}}}
$$

are forbidden by $\mathrm{SU}(3)_{f}$. Technically the second operator in Eq. (8) could be made gauge-invariant if $u^{c}$ were assigned the conjugate representation to that of $d^{c}$ and $e^{c}$, but this does not happen for the $\mathrm{SU}(3)_{f}$ model nor for the embeddings of matter into $\mathrm{SU}(5)$ or $\mathrm{SO}(10)$ representations. These operators can be made gauge-invariant by adding a pair of $\mathrm{SU}(3)_{f}$ breaking superfields $\Sigma(\mathbf{3})$ and $\bar{\Sigma}(\overline{\mathbf{3}})$, whereby Eq. (8) becomes

$$
\frac{Q Q Q L \bar{\Sigma}}{M_{\mathrm{Pl}}^{2}}, \frac{u^{c} u^{c} d^{c} e^{c} \Sigma}{M_{\mathrm{Pl}}^{2}} .
$$

Below the $\mathrm{SU}(3)_{f}$ symmetry breaking scale, these dimension- 6 operators map onto the dimension- 5 operators above with tiny coefficients of order $\langle\Sigma\rangle / M_{\mathrm{Pl}}$. This is sufficient to cure the fast proton decay problem that results from the ordinarily unsuppressed dimension- 5 operators.

A supersymmetrized version of the $\mathrm{SU}(3)_{f}$ model has even more interesting constraints. All dimension $\leq 4$ lepton number violating superpotential terms $Q L d^{c}, L L e^{c}$, and $L H_{u}$ are forbidden by $\mathrm{SU}(3)_{f}$. Again, higher dimension operators with $\mathrm{SU}(3)_{f}$ breaking fields will reintroduce these terms, but (for the first two) this leads to significant suppression. If the flavor symmetry were promoted to $\mathrm{U}(3)_{f}$, the dimension- 4 baryon number violating term $u^{c} d^{c} d^{c}$ would also be forbidden. An exact flavor symmetry could serve in precisely the same role as matter parity on superfields ( $R$-parity on fields). Of course the flavor symmetry is broken, and this reintroduces these so-called $R$-parity violating operators. It would be interesting to see if $R$-parity could be discarded in favor of a spontaneously broken $\mathrm{U}(3)_{f}$ flavor symmetry without sacrificing a long-lived proton.

In summary, an extension of the Standard Model with an $\mathrm{SU}(3)_{f}$ gauged flavor symmetry is presented that explains why there are three generations of matter and predicts the existence of three right-handed neutrinos. This argument is independent of grand unification or extra universal dimensions. The right-handed Majorana mass scale results from spontaneous $\mathrm{SU}(3)_{f}$ symmetry breaking. If the breaking scale is "low", less than of order $1000 \mathrm{TeV}$, deviations in flavor changing neutral current processes are expected due to tree-level flavor gauge boson exchange. It should be emphasized that such a Majorana mass scale is completely consistent with the see-saw explanation for neutrino mass generation so long as the Dirac masses of the neutrinos are less than but of order the muon mass. This is a perfectly reasonable possibility given that $\mathrm{SU}(3)_{f}$ has freed us from thinking only in terms of grand unification. The supersymmetric extension including a gauged $\mathrm{SU}(3)_{f}$ is straightforward. The fast proton decay problem from dimension- 5 Planck-suppressed operators is automatically cured, and certain $R$-parity violating couplings are naturally suppressed. Combining the $\mathrm{SU}(3)_{f}$ gauged flavor symmetry with models that attempt to explain the structure of the quark, lepton, or neutrino mass matrices is an extremely interesting direction left for future work.

I have benefited from discussions with B. Balantekin, V. Barger, A. Nelson, Y. Nir, G. Shiu, and L.-T. Wang. I 
also thank the CERN theory group for hospitality where part of this work was completed. This work was supported in part by the U.S. Department of Energy under contract DE-FG02-95ER40896.

[1] S. L. Adler, Phys. Rev. 177, 2426 (1969); J. S. Bell and R. Jackiw, Nuovo Cim. A 60, 47 (1969); W. A. Bardeen, Phys. Rev. 184, 1848 (1969); C. Bouchiat, J. Iliopoulos and P. Meyer, Phys. Lett. B 38, 519 (1972); D. J. Gross and R. Jackiw, Phys. Rev. D 6, 477 (1972).

[2] E. Witten, Phys. Lett. B 117, 324 (1982).

[3] L. Alvarez-Gaume and E. Witten, Nucl. Phys. B 234, 269 (1984).

[4] C. Q. Geng and R. E. Marshak, Phys. Rev. D 39, 693 (1989). J. A. Minahan, P. Ramond and R. C. Warner, Phys. Rev. D 41, 715 (1990).

[5] Y. Fukuda et al. [Super-Kamiokande Collaboration], Phys. Rev. Lett. 81, 1562 (1998) arXiv:hep-ex/9807003.

[6] Q. R. Ahmad et al. [SNO Collaboration], Phys. Rev. Lett. 89, 011301 (2002) arXiv:nucl-ex/0204008.

[7] K. Eguchi et al. [KamLAND Collaboration], Phys. Rev. Lett. 90, 021802 (2003) arXiv:hep-ex/0212021.

[8] For a review, see C. Weinheimer, arXiv:hep-ex/0210050

[9] M. Gell-Mann, P. Ramond and R. Slansky, Print-800576 (CERN); T. Yanagida, Prog. Theor. Phys. 64, 1103 (1980); R. N. Mohapatra and G. Senjanovic, Phys. Rev. Lett. 44, 912 (1980).

[10] H. Georgi, Particles and Fields, Proceedings of the
APS Division of Particles and Fields, ed C. Carlson; H. Fritzsch and P. Minkowski, Annals Phys. 93, 193 (1975).

[11] T. Yanagida, Phys. Rev. D 20, 2986 (1979); Prog. Theor. Phys. 64, 1103 (1980).

[12] F. Wilczek and A. Zee, Phys. Rev. Lett. 42, 421 (1979).

[13] T. Maehara and T. Yanagida, Prog. Theor. Phys. 61, 1434 (1979).

[14] $\mathrm{SO}(3)$ is naively better than $\mathrm{SU}(2)$, with only odd dimensional representations, but $\mathrm{SO}(3)$ with just 3's is physically indistinguishable from $\mathrm{SU}(2)$ with just 3's, and so there is no unambiguous explanation for three generations.

[15] See e.g. J. L. Chkareuli, JETP Lett. 32, 671 (1980) [Pisma Zh. Eksp. Teor. Fiz. 32, 684 (1980)]; Z. G. Berezhiani and J. L. Chkareuli, JETP Lett. 35, 612 (1982) [Pisma Zh. Eksp. Teor. Fiz. 35, 494 (1982)]; K. Tamvakis and G. Zoupanos, Phys. Lett. B 126, 314 (1983). Z. G. Berezhiani, Phys. Lett. B 129, 99 (1983); M. Soldate, M. H. Reno and C. T. Hill, Phys. Lett. B 179, 95 (1986).

[16] See e.g. M. Leurer, Y. Nir and N. Seiberg, Nucl. Phys. B 420, 468 (1994) arXiv:hep-ph/9310320.

[17] S. Dimopoulos and J. R. Ellis, Nucl. Phys. B 182, 505 (1982).

[18] T. Appelquist, H. C. Cheng and B. A. Dobrescu, Phys. Rev. D 64, 035002 (2001) arXiv:hep-ph/0012100.

[19] B. A. Dobrescu and E. Poppitz, Phys. Rev. Lett. 87, 031801 (2001) arXiv:hep-ph/0102010.

[20] S. Elitzur and V. P. Nair, Nucl. Phys. B 243, 205 (1984). 